\title{
Imaging for Pulmonary Embolism in Sickle Cell Disease: A 17-Year Experience
}

\author{
Patrick Tivnan ${ }^{1}$, Henny H. Billett ${ }^{2}$, Leonard M. Freeman ${ }^{1}$, and Linda B. Haramati ${ }^{1,3}$ \\ ${ }^{1}$ Department of Radiology, Montefiore Medical Center, Albert Einstein College of Medicine, Bronx, New York; ${ }^{2}$ Division of \\ Hematology, Department of Oncology, Montefiore Medical Center, Albert Einstein College of Medicine, Bronx, New York; and \\ ${ }^{3}$ Department of Medicine, Montefiore Medical Center, Albert Einstein College of Medicine, Bronx, New York
}

Sickle cell disease, a complex disorder with known pulmonary complications, has the potential to confound the diagnosis of pulmonary embolism. We hypothesized that when the choice of imaging is guided by chest radiographic results, CT pulmonary angiography (CTPA) and ventilation-perfusion (V/Q) scintigraphy have comparable diagnostic performance in sickle cell disease. Methods: A retrospective cohort of adults with sickle cell disease who were imaged for suspected pulmonary embolism with either CTPA or V/Q, from 2000 to 2016 at our institution, was established. To reduce radiation exposure, our practice recommends $V / Q$ for stable patients with normal chest radiographs. Results of index pulmonary embolism imaging, 90-d follow-up, and results of chest radiography were recorded. Results: Two hundred forty-five adults with sickle cell disease comprised the cohort. The mean age ( $\pm S D)$ was $33 \pm 10.5 y$, and $58 \%$ (141) were men. Index imaging was V/Q in $62.9 \%(n=154)$ and CTPA in $37.1 \%(n=91)$. Chest radiographs, performed in $96.3 \%(n=236)$, were normal in $72.9 \%(n=172)$. Imaging results for pulmonary embolism were negative in $88.2 \%$ ( $n=216)$, positive in $4.1 \%(n=10)$, and indeterminate in $7.8 \%$ $(n=19)$ with no difference between V/Q and CTPA $(P=0.63)$. Reimaging within $90 \mathrm{~d}$ occurred in $9.8 \%(n=24), 14.7 \%(20 / 136)$ after initial V/Q, and 5\% (4/109) after initial CTPA ( $P=0.08)$. Reimaging revealed a pulmonary embolism diagnosis after negative/ indeterminate results in $0.7 \%(1 / 149)$ of $\mathrm{V} / \mathrm{Qs}$ and $1.2 \%$ of $(1 / 86)$ CTPAs $(P=0.69)$. Over the $17-y$ study period, $47 \%(114 / 245)$ underwent repeated imaging, and $11 \%(27 / 245)$ were diagnosed with pulmonary embolism at least once. Conclusion: In sickle cell disease patients with suspected pulmonary embolism, positive imaging rates were low for any given clinical presentation, but $11 \%$ of the cohort was diagnosed with pulmonary embolism over the 17-y study period. CTPA and V/Q performed comparably for pulmonary embolism diagnosis when the choice of imaging was guided by results of chest radiography. Hence, $V / Q$ is a reasonable first choice for sickle cell disease patients with normal chest radiographs.

Key Words: sickle cell disease; pulmonary embolism; scintigraphy lung; ventilation perfusion; computed tomography pulmonary angiogram

J Nucl Med 2018; 59:1255-1259

DOI: 10.2967/jnumed.117.205641

Received Nov. 19, 2017; revision accepted Dec. 30, 2017.

For correspondence or reprints contact: Linda B. Haramati, Department of Radiology, Montefiore Medical Center, Albert Einstein College of Medicine, 111 East 210 St., Bronx, NY 10467.

E-mail: Iharamati@gmail.com

Published online Feb. 1, 2018.

COPYRIGHT (C 2018 by the Society of Nuclear Medicine and Molecular Imaging.
$\mathbf{T}$ he differentiation of acute pulmonary embolism from pneumonia, fluid overload, and chronic pulmonary hypertension in individuals with sickle cell disease is challenging. It can be even more difficult to distinguish the clinical features of thromboembolic pulmonary embolism from other complications such as bone marrow/fat embolism, in situ pulmonary artery thrombosis, and acute chest syndrome. Indeed, upward of $90 \%$ of sickle cell disease adults have abnormal pulmonary function tests (1), which may be related to the increased rates of pulmonary fibrosis, pulmonary hypertension, pulmonary hemosiderosis, and asthma (2-4). These additional pathologies have the potential to clinically mask the presence of pulmonary embolism, delaying or obstructing management even further. As sickle cell disease pulmonary complications often have different treatments, therapeutic endpoints, and prognoses, their distinction is necessary for appropriate management.

In patients with sickle cell disease suspected of having a pulmonary embolism, lung imaging is often the first test used because D-dimer results may be elevated from other causes (5). The 2 most commonly used pulmonary embolism imaging tests are the CT pulmonary angiogram (CTPA) and ventilation-perfusion (V/Q) scintigraphy. Over the past several decades, CTPA has become the most commonly used imaging test for pulmonary embolism. Although less commonly performed than CTPA, the $\mathrm{V} / \mathrm{Q}$ scan is a reasonable, and currently a lower radiation dose imaging modality, for suspected pulmonary embolism (6-9). Reducing radiation exposure is particularly relevant to sickle cell disease patients who are younger and thus more biologically susceptible to the ill effects of radiation exposure, and whose lifelong chronic illness makes them both more likely to develop significant kidney disease and to undergo repeated imaging (5). The V/Q scan also has the advantage of not requiring the administration of intravenous contrast.

Although studies have compared the performance characteristics of CTPA and the V/Q scan for pulmonary embolism in the general population, to our knowledge, no studies to date have compared their diagnostic performance in individuals with sickle cell disease. There has been clinical concern that other sickle cell disease-related pulmonary pathologies could affect the diagnostic performance of $\mathrm{V} / \mathrm{Q}$. On the other hand, the physiologic information provided by V/Q may provide added value.

Our institution's practice is to use the chest radiograph to triage stable patients with normal chest radiographs to V/Q scan and those with abnormal radiographs to CPTA; patients with contraindications to either examination had the alternative examination recommended. Final imaging decision remains in the hands of the 
requesting physician (10). We hypothesized that CTPA and V/Q scintigraphy would have comparable diagnostic performance, with this practice algorithm, for individuals with sickle cell disease imaged for pulmonary embolism.

\section{MATERIALS AND METHODS}

\section{Patient and Imaging Selection}

The population for this 17-y single-center retrospective cohort study was derived from a combination of databases and patient-level chart review. A database of sickle cell disease adults aged $18 \mathrm{y}$ or older seen at Montefiore Medical Center, Bronx, New York, between 2000 and 2016 established the cohort. Sickle cell disease patients could be $\mathrm{HgSS}, \mathrm{HgSC}, \mathrm{HgS}^{\circ}, \mathrm{HgSD}, \mathrm{HgSE}$, or $\mathrm{HgSO}$, or $\mathrm{HgS}^{+}$ Thalassemia. Sickle cell disease was confirmed by hemoglobin electrophoresis results. Patients were excluded if the hemoglobin electrophoresis was absent or inconsistent with the disease or the clinical notes did not support the diagnosis. Patients with confirmed sickle cell disease who were imaged with V/Q scan or CTPA between 2000 and 2016 were identified with Montage Health Care Solution system and Looking Glass Clinical Analytics (Streamline Health), which integrates clinical and administrative datasets. The date of first imaging with either V/Q scan or CTPA during the study period was considered the index date. Follow-up V/Q scans and CTPAs performed after the index date were noted. In the 3 cases for which individuals received bone marrow transplantations during this period, only those studies performed before the bone marrow transplantation were included.

This study was approved by the Einstein Montefiore Institutional Review Board (\#2016-6115), which waived informed consent.

\section{Data Collection}

Demographics including age at the index scan, sex, and sickle cell hemoglobinopathy type were recorded as was patient location, when available.

During the study period, CTPAs were performed on a variety of helical scanners ranging from single-detector to 64-detector row CT technology. Radiation dosimetry data for the present study cohort were not collected. However, institutional dosimetry data for CTPA at our institution, available from 2007 to $2015(11,12)$, demonstrated decreased radiation exposure from CTPA from 11 to $5.3 \mathrm{mSv}$, more recently. The V/Q scan technique was stable during the study period, with an estimated radiation exposure of $2.6 \mathrm{mSv}$ (13). The ventilation portion routinely used $1.48 \mathrm{GBq}(40 \mathrm{mCi})$ of aerosolized ${ }^{99 \mathrm{~m}} \mathrm{Tc}-$ labeled DTPA followed by the perfusion scan performed after intravenous administration of $1.11-1.48 \mathrm{GBq}(3-4 \mathrm{mCi})$ of ${ }^{99 \mathrm{~m}} \mathrm{Tc}-$ labeled macroaggregated albumin.

For each scan, the relevant information was ascertained from the chart and official imaging report including the type and date of the study, the imaging result with regard to pulmonary embolism, and the interpretation of the preceding radiograph, when available. Chest radiographs were classified as normal or abnormal based on whether the report described pulmonary or pleural findings such as effusions, infiltrates, or opacities. Each V/Q or CTPA study was characterized on the basis of the clinical report as being negative, positive, or indeterminate for pulmonary embolism. CTPAs indicating central, segmental, or subsegmental pulmonary embolism were considered positive. A study was considered indeterminate if it was explicitly interpreted as indeterminate or nondiagnostic or the report impression was uncertain with regard to pulmonary embolism. Those images positive for pulmonary embolism were further divided by whether the result indicated acute or chronic pulmonary embolism. For V/Q scans, probability interpretation was used until mid-2009 when we adopted the trinary interpretation scheme. Probability interpretations were reclassified into trinary interpretation as we previously described (14).
A prior V/Q scan indicating a mismatched defect in the same location was used to diagnose chronic pulmonary embolism.

For positive imaging studies, additional information was ascertained, if available, including results of venous ultrasounds for deep vein thrombosis, presence of vascular access devices, and therapeutic anticoagulation during the hospitalization and a discharge prescription.

\section{Statistical Analysis}

Descriptive statistics were used for patient characteristics, rates of pulmonary embolism detection in relationship to the index scan, and characteristics of positive pulmonary embolism imaging. The $\chi^{2}$ or Fisher exact test was used, as appropriate, with significance at a $P$ value of less than $0.05 . \chi^{2}$ statistics were used for bivariate analyses of the differences between CTPA and V/Q scan results and their relations with preceding results of chest radiography. Statistical significance was defined as a $P$ value of less than 0.05 .

\section{RESULTS}

\section{Patient Characteristics and Index Imaging}

The cohort comprised 245 adults with sickle cell disease. Mean age was $33 \pm 10.5 \mathrm{y}$ and $58 \%$ (141) were men (Table 1). Index imaging was V/Q scan in $62.9 \%(154 / 245)$ and CTPA in $37.1 \%$ (91/245). As shown in Table 2, 96.3\% (236/245) of the cohort underwent a chest radiograph before their index scan and the results were normal in $72.9 \%$ (172/236). Overall, the scans were negative for pulmonary embolism in $88.2 \%$ (216/245), positive for pulmonary embolism in $4.1 \%$ (10/245), and indeterminate in $7.8 \%$ (19/245). Indeterminate results were significantly less common in the more recent era, from 2010 to 2016, compared with the earlier era (2000-2009) (4.8\% [8/165] vs. 12.7\% [10/79], respectively, $P=0.04)$. This decrease in indeterminate results in the recent era occurred for both modalities, statistically significantly for CTPA (CTPA: $1.6 \%$ [1/64] vs. $18.5 \%$ [5/27], $P=0.008$; V/Q: $6.9 \%$ [7/ $101]$ vs. $9.6 \%[5 / 52], P=0.54)$. There was overall no difference between V/Q scan and CTPA with regards to pulmonary embolism negative, positive, or indeterminate results $(P=0.63)$, when the chest radiograph was normal $(P=84)$, or abnormal $(P=0.57)$.

Among the 10 patients with acute pulmonary embolism on index imaging, all were anticoagulated as inpatients, and $90 \%$ (9/10) were discharged on anticoagulation. One patient, who left

TABLE 1

Study Cohort Characteristics

\begin{tabular}{|c|c|}
\hline Characteristic & $n=245$ \\
\hline Mean age \pm SD $(y)$ & $33 \pm 10.5$ \\
\hline Male & $141 / 245(57.5 \%)$ \\
\hline \multicolumn{2}{|l|}{ Hemoglobin type } \\
\hline Sickle S & $182(74.3 \%)$ \\
\hline Sickle C & $40(16.3 \%)$ \\
\hline S- $\beta$-Thalassemia & $21(8.6 \%)$ \\
\hline Sickle, other* & $2(0.8 \%)$ \\
\hline \multicolumn{2}{|l|}{ Patient location } \\
\hline Emergency department & $60 / 245(24.5 \%)$ \\
\hline Inpatient & $104 / 245(42.4 \%)$ \\
\hline Unknown & $81 / 245$ (33\%) \\
\hline
\end{tabular}

${ }^{*} 1$ patient with HgSE, 1 patient with $\mathrm{HgS}-\mathrm{O}$ Arab. 
TABLE 2

Results of Index Imaging for Pulmonary Embolism

\begin{tabular}{|c|c|c|c|c|c|}
\hline Index scan & Imaging test & PE- (216 [88.2\%]) & $\mathrm{PE}+(10[4.1 \%])$ & $\begin{array}{c}\text { Indeterminate } \\
(19 \text { [7.8\%]) }\end{array}$ & $P$ \\
\hline \multirow[t]{2}{*}{ All $(n=245)^{*}$} & CTPA $(n=91)$ & $80(87.9 \%)$ & $5(5.5 \%)$ & $6(6.6 \%)$ & 0.63 \\
\hline & $\mathrm{V} / \mathrm{Q}(n=154)$ & $136(88.3 \%)$ & $5(3.2 \%)$ & $13(8.4 \%)$ & \\
\hline \multirow[t]{2}{*}{ Normal $(n=172)$ chest radiograph } & CTPA $(n=40)$ & $37(92.5 \%)$ & $1(2.5 \%)$ & $2(5.0 \%)$ & 0.84 \\
\hline & $\mathrm{V} / \mathrm{Q}(n=132)$ & $118(89.4 \%)$ & $4(3.0 \%)$ & $10(7.6 \%)$ & \\
\hline \multirow[t]{2}{*}{ Abnormal $(n=64)$ chest radiograph } & CTPA $(n=47)$ & $39(80.8 \%)$ & $4(8.8 \%)$ & $4(8.5 \%)$ & 0.57 \\
\hline & $\mathrm{V} / \mathrm{Q}(n=17)$ & 13 (76.5\%) & 1 (5.9\%) & 3 (17.7\%) & \\
\hline
\end{tabular}

${ }^{*}$ Nine patients did not undergo chest radiographs.

$\mathrm{PE}=$ pulmonary embolism.

against medical advice, did not accept anticoagulation at discharge. A potential source for the pulmonary embolism was evident in $50 \%$ (5/10): 3 of 5 had deep vein thrombosis on ultrasound, and 2 of 5 had a vascular access device in situ.

\section{Repeated Imaging}

Of the study population, 9.8\% (24/245) underwent a second imaging study within $90 \mathrm{~d}$ of the index examination (Table 3); results were concordant in $54.2 \%$ (13/24). Patients with index V/Q underwent more second imaging examinations than those with index CTPA, $52.6 \%$ versus $36.2 \%(P=0.01)$. Among the $7.8 \%$ $(19 / 245)$ patients with initial indeterminate results, $47 \%(9 / 19)$ underwent reimaging within $90 \mathrm{~d}$. Of the indeterminate V/Q scans, 7 of 9 were reimaged within $3 \mathrm{~d}$ by CTPA; 6 were negative and 1 was diagnosed as a chronic pulmonary embolism. One patient with index CTPA was reimaged by V/Q on day 7, with results positive for pulmonary embolism; this patient was discharged on new anticoagulation. For 1 patient with a history of deep vein thrombosis and pulmonary embolism, the initial indeterminate V/Q scan was followed but a subsequent V/Q scan that demonstrated chronic pulmonary embolism and the patient was maintained on anticoagulation. Among the 10 patients with indeterminate scans who were not reimaged within $90 \mathrm{~d}, 2$ were maintained on anticoagulation for prior known thromboembolic disease, 4 with low clinical suspicion were discharged without anticoagulation, 1 was started on new anticoagulation, and 3 had documentation that was unclear.

Although additional imaging was more likely when the index examination was positive or indeterminate for both CTPA and

TABLE 3

90-Day V/Q and CTPA Comparison $(n=24)$

\begin{tabular}{llcc}
\hline \multicolumn{1}{c}{ First scan result $(n)$} & Result of repeated scan & CTPA & 2 \\
\hline V/Q negative (8) & Negative & 4 & 0 \\
& Positive & 1 (chronic PE) & 0 \\
V/Q indeterminate (8) & Other & 6 & 0 \\
& Negative & 0 & 0 \\
V/Q positive (4) & Positive & 1 (indeterminate) & 1 (chronic PE) \\
& Other & 1 & 0 \\
& Negative & 3 & 0 \\
CTPA negative (3) & Positive & 0 & 0 \\
& Other & 2 & 1 \\
CTPA indeterminate (1) & Negative & 0 & 0 \\
& Positive & 0 & 0 \\
& Other & 0 & 0 \\
& Negative & 0 & 0
\end{tabular}

${ }^{*} \mathrm{~V} / \mathrm{Q}$ repeated $57 \mathrm{~d}$ after initial V/Q on another hospitalization. Subsequent CTPA performed during that hospitalization was reported as negative for pulmonary embolism; patient was discharged without anticoagulation.

${ }^{\dagger}$ Patient already on anticoagulation for prior deep vein thrombosis.

$\mp \mathrm{V} / \mathrm{Q}$ performed on day 7 , and patient discharged on anticoagulation.

$\mathrm{PE}=$ pulmonary embolism. 
V/Q scan $(P<0.01$, both), $4.6 \%$ (10/216) with negative index imaging also underwent repeated imaging within $90 \mathrm{~d}$. These results were again negative for acute pulmonary embolism in $90 \%(9 / 10)$.

Over the 17-y study period, repeated imaging was common. Of the 245 patients, $36.7 \%(90 / 245)$ underwent at least 1 additional imaging study for pulmonary embolism more than $90 \mathrm{~d}$ after the index scan, during a different clinical presentation. Among patients with an index CTPA, 36.2\% (33/91) underwent a second imaging examination at any point within the study period versus $52.6 \%(81 /$ 154) for those with index V/Q $(P=0.01)$. Among the $47 \%(114 /$ $245)$ of the study population who underwent imaging for pulmonary embolism more than once, $32 \%(78 / 245)$ had 2 studies, $7.8 \%$ (19/ 245) had 3 studies, and 7\% (17/245) had 4 or more studies, with 1 patient undergoing 21 scans (18 V/Q scans and 3 CTPA) (Table 4). Of the study population, $11 \%(27 / 245)$ had imaging positive for pulmonary embolism at least once over the $17-y$ study period.

\section{DISCUSSION}

This is the first study, to our knowledge, that has addressed the performance of CTPA and V/Q scan for suspected pulmonary embolism in patients with sickle cell disease. We found comparable diagnostic performance for the 2 modalities in our practice. We observed a low prevalence of pulmonary embolism: only $4 \%$ for a given clinical presentation, lower than previously reported (15-17). However, over the 17 -y study period, $11 \%$ of our sickle cell disease cohort who presented with symptoms that warranted imaging had a diagnosis of pulmonary embolism.

Our clinical practice recommends V/Q scans for stable patients with normal chest radiographs and for those with contraindications to CTPA and CTPA for those with abnormal chest radiograph or unstable patients. This practice has been validated in the general emergency department population (10) and is designed to refer suitable patients for V/Q scans, which typically performs well in those with normal chest radiograph and currently confers a lower radiation exposure than CTPA $(7,13)$. However, V/Q scans may have diagnostic limitations in the presence of lung disease; the practice of referring patients with abnormal chest radiographs to

TABLE 4

17-Year Pulmonary Embolism Imaging of Cohort $(n=245)$

\begin{tabular}{|lc}
\hline \multicolumn{1}{c}{ Imaging test } & Number (5) \\
\hline V/Q scans & 318 \\
\hline CTPA & 187 \\
\hline Patients with only V/Q & $107(43.7 \%)$ \\
\hline Mean number of V/Q & 1.5 \\
\hline Maximum number V/Q & 5 \\
\hline Patients with only CTPA & $65(26.5 \%)$ \\
\hline Mean number of CTPA & 1.2 \\
\hline Maximum number CTPA & 4 \\
\hline Patients with both CTPA and V/Q scans & $73(29.8 \%)$ \\
\hline Mean number CTPA & 1.5 \\
\hline Maximum number CTPA & 5 \\
\hline Mean number V/Q & 2.0 \\
\hline Maximum number V/Q & 18 \\
\hline
\end{tabular}

CTPA mitigates these limitations and provides diagnostic alternatives demonstrated on CT.

An important hypothetical concern regarding the performance of V/Q scans in patients with sickle cell disease, in particular, relates to the common pulmonary vascular complications including vasoocclusive crisis, in situ pulmonary artery thrombosis, and fat/bone marrow embolism due to bone infarction (18). We were unsure whether these complications of sickle cell disease might result in high false-positive and indeterminate rates for V/Q scans compared with CTPA. Our results, however, do not substantiate this concern; most of both V/Q scans and CTPAs were negative for pulmonary embolism and the positivity rate of V/Q scans and CTPA were comparable. Although the indeterminate rate for V/Q was higher than for CTPA, this difference did not reach statistical significance and was most notable for patients with abnormal chest radiographic results who likely underwent evaluation with a V/Q scan, in exception to our clinical algorithm, due to contraindications to CTPA. In addition, imaging results were significantly less frequently indeterminate in the recent era, 2010-2016, compared with 2000-2009, statistically significantly so for CTPA. During this time, we adopted the trinary interpretation scheme for V/Q (14) and began to question the clinical implications of limited negative CTPA results (19). Among the 19 patients with indeterminate results, anticoagulation was initiated in only 2 patients after indeterminate CTPAs. Hence, clinical outcomes were indistinguishable between patients who underwent index CTPA and index V/Q scan.

Sickle cell disease is often described as causing a hypercoagulable state due to several mechanisms including decreased levels of anticoagulation factors, upregulation of endothelial procoagulant activity, coagulation factor and platelet activation, increased cytokine expression, decreased nitric oxide, and chronic damage from sickling $(20,21)$. Previous reports have suggested that this prothrombotic diathesis has resulted in an increased rate of large vessel stroke, increased venous thromboembolism during pregnancy, and pulmonary embolism $(22,23)$. Stein et al. and Novelli et al. both noted a significantly increased rate of pulmonary embolism in admitted sickle cell disease patients compared with hemoglobin A controls based on diagnosis at discharge $(15,17)$. Another study by Mekontso et al. evaluated the rates of pulmonary embolism in individuals with sickle cell disease who were hospitalized for acute chest syndrome and found pulmonary embolism in $17 \%$ (16).

In contrast, we have previously reported pulmonary embolism rates of $13.5 \%$ for CTPA and 3.5\% for V/Q scan in our general emergency department population (10). Although cohort characteristics differ between the 2 groups, the rate of pulmonary embolism in sickle cell disease did not exceed the population for whom a diagnosis of pulmonary embolism is considered. One feature that is consistent in the studies evaluating pulmonary embolism in sickle cell disease is the failure to demonstrate an increased rate of deep vein thrombosis in the population. This suggests that the excess diagnosis of pulmonary embolism in sickle cell disease may not be related to typical thromboembolic disease but perhaps due to other pulmonary vascular complications such as fat emboli or local nonembolic vasoocclusion. In contrast to our study, which included emergency department as well as inpatients, prior studies solely included inpatients-a population with a greater illness burden and with increased exposure to vascular access devices/ intravenous lines; these may represent secondary prothrombotic risks that are not unique to the sickle cell disease population.

Radiation exposure from medical imaging has grown substantially in the United States over the past several decades. This 
issue is especially important for young people who are particularly vulnerable to the biologic hazards of radiation exposure and for patients with chronic conditions (24). Because of advances in medical therapy, sickle cell disease individuals are enjoying longer life expectancies and better health $(25,26)$ but still require a high level of ongoing care for the many potential complications, including renal dysfunction. Appropriate diagnostic evaluation for these complications will often involve imaging. The good diagnostic performance of the V/Q scan in sickle cell disease patients in our practice confers a radiation reduction advantage and obviates the need for iodinated intravenous contrast. Even with aggressive radiation reduction strategies, the radiation exposure for 64-detector row CTPA scanners we - and many other institutions-currently use is double to triple that of V/Q scans (11-13). When more advanced CT technologies become widespread, this difference may be mitigated and then a further reassessment of risk-benefit recommendations will be necessary.

When evaluating pulmonary embolism imaging, nearly half of our study population was imaged more than once and one of our patients underwent 21 separate pulmonary embolism imaging studies over the 17-y period. Although not addressed in the present study, many of the study patients also underwent imaging for multiple other indications. Therefore, clinically appropriate radiation reduction strategies should continue to be factored into clinical decision making for this vulnerable population.

The main limitation in this study is its retrospective design, resulting in the inherent limitations related to data available in the documented records. Because this study spanned $17 \mathrm{y}$, there was more information available in the medical record for recent cases. However, this is unlikely to bias our results toward a particular imaging modality. We did not study the relationship between choice of imaging modality and alternative diagnoses in our sickle cell disease cohort nor did we gather data regarding adverse outcomes related to use of iodinated contrast agents. Moreover, the clinical algorithm using chest radiographs to direct patients with suspected pulmonary embolism to be imaged with V/Q scan or CTPA limits the generalizability of these results to other populations. In addition, we were unable to account for the complexity related to ongoing changes in technology, new insights in radiation biology, and our increasing understanding of the unique data conveyed by physiologic versus anatomic imaging.

\section{CONCLUSION}

Sickle cell disease patients with suspected pulmonary embolism demonstrated low positive imaging rates for a given clinical presentation, but $11 \%$ of the cohort was diagnosed with pulmonary embolism over the 17-y study period. CTPA and V/Q performed comparably for pulmonary embolism diagnosis when the choice of imaging was guided by results of chest radiography. Hence, V/Q is a reasonable first choice for sickle cell disease patients with normal chest radiographs.

\section{DISCLOSURE}

Dr. Leonard M. Freemen is on the scientific advisory board of Jubilant Draximage Corp. Linda B. Haramati's spouse is a board member of Kryon, LTD. No other potential conflict of interest relevant to this article was reported.

\section{ACKNOWLEDGMENT}

This paper was accepted for presentation American Society of Hematology Annual Meeting, Atlanta, Georgia, December 11, 2017.

\section{REFERENCES}

1. Klings ES, Wyszynski DF, Nolan VG, Steinberg MH. Abnormal pulmonary function in adults with sickle cell anemia. Am J Respir Crit Care Med. 2006;173:1264-1269.

2. Gladwin MT, Vichinsky E. Pulmonary complications of sickle cell disease. N Engl J Med. 2008;359:2254-2265.

3. Powars D, Weidman JA, Odom-Maryon T, Niland JC, Johnson C. Sickle cell chronic lung disease: prior morbidity and the risk of pulmonary failure. Medicine (Baltimore). 1988;67:66-76.

4. Knight-Madden JM, Forrester TS, Lewis NA, Greenough A. Asthma in children with sickle cell disease and its association with acute chest syndrome. Thorax. 2005;60:206-210.

5. Francis RB Jr. Elevated fibrin D-dimer fragment in sickle cell anemia: evidence for activation of coagulation during the steady state as well as in painful crisis. Haemostasis. 1989;19:105-111.

6. Sostman HD, Stein PD, Gottschalk A, Matta F, Hull R, Goodman L. Acute pulmonary embolism: sensitivity and specificity of ventilation-perfusion scintigraphy in PIOPED II study. Radiology. 2008;246:941-946.

7. Anderson DR, Kahn SR, Rodger MA, et al. Computed tomographic pulmonary angiography vs ventilation-perfusion lung scanning in patients with suspected pulmonary embolism: a randomized controlled trial. JAMA. 2007;298:2743-2753.

8. Waxman AD, Bajc M, Brown M, et al. Appropriate use criteria for ventilationperfusion imaging in pulmonary embolism: summary and excerpts. J Nucl Med. 2017;58(5):13N-15N.

9. Parker MS, Hui FK, Camacho MA, Chung JK, Broga DW, Sethi NN. Female breast radiation exposure during CT pulmonary angiography. AJR. 2005;185:1228-1233.

10. Stein EG, Haramati LB, Chamarthy M, Sprayregen S, Davitt MM, Freeman LM. Success of a safe and simple algorithm to reduce use of CT pulmonary angiography in the emergency department. AJR. 2010;194:392-397.

11. Rawat U, Cohen SL, Levsky JM, Haramati LB. ACR white paper-based comprehensive dose reduction initiative is associated with a reversal of the upward trend in radiation dose for chest CT. J Am Coll Radiol. 2015;12:1251-1256.

12. Hsu KA, Levsky JM, Haramati LB, Gohari A. Performance of a simple robust empiric timing protocol for CT pulmonary angiography. Clin Imaging. 2017;48:17-21.

13. Schembri GP, Miller AE, Smart R. Radiation dosimetry and safety issues in the investigation of pulmonary embolism. Semin Nucl Med. 2010;40:442-454.

14. Glaser JE, Chamarthy M, Haramati LB, Esses D, Freeman LM. Successful and safe implementation of a trinary interpretation and reporting strategy for V/Q lung scintigraphy. J Nucl Med. 2011;52:1508-1512.

15. Stein PD, Beemath A, Meyers FA, Skaf E, Olson RE. Deep venous thrombosis and pulmonary embolism in hospitalized patients with sickle cell disease. Am J Med. 2006;119:897.e97-897.e11.

16. Mekontso Dessap A, Deux JF, Abidi N, et al. Pulmonary artery thrombosis during acute chest syndrome in sickle cell disease. Am J Respir Crit Care Med. 2011;184:1022-1029.

17. Novelli EM, Huynh C, Gladwin MT, Moore CG, Ragni MV. Pulmonary embolism in sickle cell disease: a case-control study. J Thromb Haemost. 2012;10:760-766.

18. Paul RN, Castro OL, Aggarwal A, Oneal PA. Acute chest syndrome: sickle cell disease. Eur J Haematol. 2011;87:191-207.

19. Yu S, Nayak GK, Levsky JM, Haramati LB. CT pulmonary angiography: clinical implications of a "limited negative" examination. JAMA Intern Med. 2015;175:447-449.

20. Naik RP, Streiff MB, Haywood C Jr, Nelson JA, Lanzkron S. Venous thromboembolism in adults with sickle cell disease: a serious and under-recognized complication. Am J Med. 2013;126:443-449.

21. Solovey A, Lin Y, Browne P, Choong S, Wayner E, Hebbel RP. Circulating activated endothelial cells in sickle cell anemia. N Engl J Med. 1997;337:1584-1590.

22. Ohene-Frempong K, Weiner SJ, Sleeper LA, et al. Cerebrovascular accidents in sickle cell disease: rates and risk factors. Blood. 1998;91:288-294.

23. Villers MS, Jamison MG, De Castro LM, James AH. Morbidity associated with sickle cell disease in pregnancy. Am J Obstet Gynecol. 2008;199:125.e1-125.e5.

24. Stein EG, Haramati LB, Bellin E, et al. Radiation exposure from medical imaging in patients with chronic and recurrent conditions. J Am Coll Radiol. 2010;7:351-359.

25. Quinn CT, Rogers ZR, McCavit TL, Buchanan GR. Improved survival of children and adolescents with sickle cell disease. Blood. 2010;115:3447-3452.

26. Lanzkron S, Strouse JJ, Wilson R, et al. Systematic review: hydroxyurea for the treatment of adults with sickle cell disease. Ann Intern Med. 2008;148:939-955. 